\title{
The Ultrasonic/Sonic Driller/Corer (USDC) as a Subsurface Drill, Sampler and Lab-On-A-Drill for Planetary Exploration Applications
}

\author{
Y. Bar-Cohen, Z. Chang, S. Sherrit, M. Badescu, and X. Bao, \\ Jet Propulsion Laboratory (JPL)/Caltech, Pasadena, California, USA, E-mail yosi@jpl.nasa.gov \\ Web: http://ndeaa.jpl.nasa.gov
}

The search for existing or past life in the Universe is one of the most important objectives of NASA's mission. For this purpose, effective instruments that can sample and conduct in-situ astrobiology analysis are being sought. In support of this objective, a novel Ultrasonic/Sonic Driller/Corer (USDC) based mechanism has been developed to probe and sample rocks, ice and soil. The USDC consists of an ultrasonic actuator that impacts a coring or drilling bit at sonic frequencies through the use of an intermediate free-mass. The USDC can produce both a core and powdered cuttings as well as emit elastic waves into the penetrated medium. For planetary exploration, this mechanism has the important advantage of requiring low axial force, virtually no torque, and can be duty cycled to require low average power. This low axial load advantage overcomes a major limitation of planetary sampling in low gravity environments and when operating from lightweight robots and rovers. The low average power operation produces a minimum temperature rise which is important for the acquisition of biologically meaningful samples. The development of the USDC is being pursued on various fronts ranging from analytical modeling to improvements of the mechanism while seeking a wide range of applications. In this paper, the latest status of the USDC development and applications that are underway is reviewed and discussed.

Keywords: Sampling, drilling, Ultrasonic/Sonic Driller/Corer (USDC), URAT, Subsurface exploration

\section{INTRODUCTION}

Future NASA exploration missions have the objective of in-situ sampling and analysis of various planets in the solar system (including Mars, Europa, Titan, comets and asteroids) and the rest of the Universe as well as possibly bringing samples to Earth for further studies. For this purpose, effective instruments that can sample and conduct in-situ astrobiology analysis are being sought. Existing drilling techniques are limited by the need for large axial forces and holding torques, high power consumption and inability to efficiently duty cycle, and they also require heavy equipment. To address these limitations, the JPL's Advanced Technologies Group [http://ndeaa.jpl.nasa.gov] and engineers from Cybersonics, Inc. jointly developed the USDC [Bao et al., 2003; http://ndeaa.jpl.nasa.gov/nasa-nde/usdc/usdc.htm]. Following the development of this novel mechanism (see Figure 1) the team conceived many innovative designs that were disclosed in NASA New Technology Reports and patents [Bar-Cohen et al. 1999; Bar-Cohen et al. 2001; Dolgin et al. 2001a; Dolgin et al 2001b; Sherrit et al. 2001; Bar-Cohen et al 2002; Sherrit et al 2002; Bar-Cohen et al. 2003a; Sherrit et al. 2003a; Bar-Cohen et al. 2003; BarCohen et al. 2003c; Bao et al. 2004]. The USDC requires low axial force, thereby overcoming one of the major limitations of planetary sampling using conventional drills in low gravity environments. This capability offers users on earth the advantage of being able to perform tough tasks of drilling and coring in hard rocks, concrete, ice and packed soil using relatively small force and relatively lightweight hardware. The USDC was demonstrated so far to: 1) drill ice and various rocks including granite, diorite, basalt and limestone, 2) not require bit sharpening, 3 ) operate at low and high temperatures, and 4) operate at low average power using duty cycling. This novel drill is capable of high-speed drilling ( 2 to $20-\mathrm{mm} / \mathrm{Watt} \cdot \mathrm{hr}$ for a $2.85 \mathrm{~mm}$ diameter bit) in basalt and Bishop Tuff using low axial preload $(<10 \mathrm{~N})$ and low average power $(<12 \mathrm{~W})$. The USDC has drilled $25-\mathrm{mm}$ deep, 6-mm diameter holes in basalt in a little over 2-hrs from a 4-kg platform using $10 \mathrm{~W}$ average and $25 \mathrm{~W}$ peak power. It has also drilled 15-cm deep, 5-mm diameter holes in sandstone in just over an hour using similar power as for the basalt drilling. The capabilities that are currently being investigated include probing the ground to select sampling sites, collecting various forms of samples (including cores and powdered cuttings), sampling long cores of hard basalt using low power and hosting sensors for measuring various properties. A series of modifications of the USDC basic configuration were made resulting in the development of the Ultrasonic/sonic Rock Abrasion Tool (URAT), Ultrasonic/Sonic Gopher for deep drilling, and the Lab-on-a-drill and many others.

The USDC is made of three key components: actuator, free-mass and bit (see Figure 1) [Bao et al, 2003]. The actuator operates as a hammering mechanism that hits the free-mass and in turn the bit is hit to fracture the rock 
that is in contact with the bit. The actuator consists of a piezoelectric stack with backing for forward power delivery and a horn for amplification of the induced displacement. The USDC is actuated by a piezoelectric stack that is driven in resonance and is held in compression by a stress bolt that prevents its fracture during operation. In the basic design the piezoelectric stack has a resonance frequency of about $20-\mathrm{kHz}$. Unlike typical ultrasonic drills where the bit is acoustically coupled to the horn, in the USDC the actuator drives a free flying mass (free-mass), which bounces between the horn tip and the drilling/coring bit converting the ultrasonic impacts to hammering at sonic frequencies. The impacts of the free-mass create stress pulses that propagate to the interface of the bit and the rock onto which the USDC is placed in contact. The rock is fractured when its ultimate strain is exceeded at the rock/bit interface.
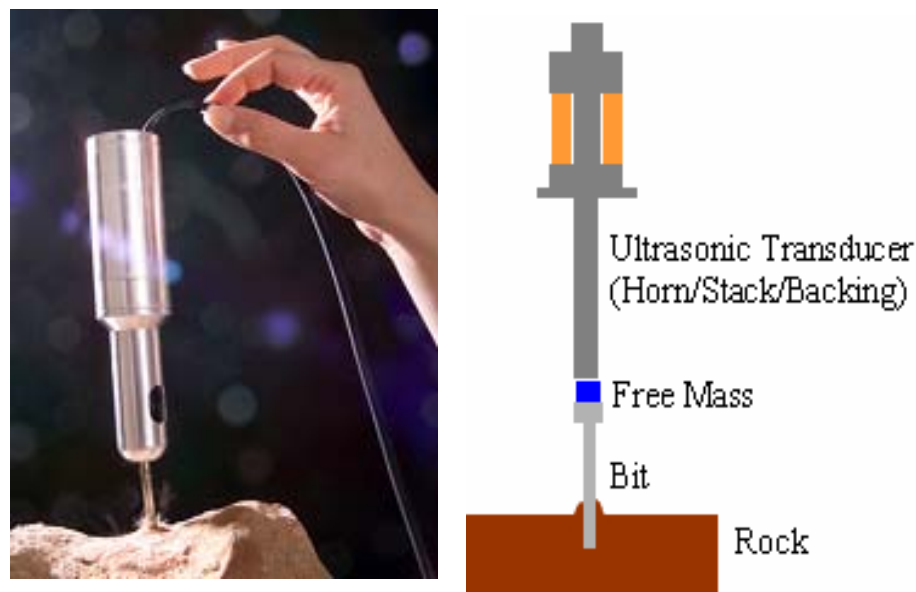

FIGURE 1: A photographic view of the USDC showing its ability to core with minimum axial force (left), and a schematic diagram of its cross-section (right).

\section{ANALYTICAL MODELING THE OPERATION OF THE USDC}

Coring via the USDC mechanism involves rock fracture under impact loading (percussion). To better understand the fracture of rocks under this impact loading, a finite element model using ANSYS (a finite element software package) was developed to investigate the propagation of the induced stress. Results were derived by assuming that the rock medium is made of isotropic material with a Young's modulus of $10 \mathrm{GPa}$ and Poisson's ratio of 0.3 . Examples of data for various soil and rock types and the related range of Young's modulus are listed in Table 1. Contour maps of the maximum principal strain were plotted and used to indicate the areas where the rock is fractured and to determine how the elastic waves propagate in the rock prior to fracture. This analytical capability allows for an estimate of the limitations on the diameter of the cored material that maintains structural integrity. Using this analysis, it is estimated that the minimum diameter of intact cores that can be produced is about 4-5 mm for medium to hard materials. The drilling rates in various rocks at $10-\mathrm{W}$ average power were calculated and a graph is shown in Figure 1 for various rock stiffness values. This capability to predict the performance of the drill allows optimizing the design of effective USDC units.

\section{LAB-ON-A-DRILL}

The USDC characteristics of using a bit that does not turn allows using it for probing and sensing in addition to sampling cores and dust. The sonic and ultrasonic hammering action on the bit allows using it as a sounder for probing the drilled medium. Further, the longitudinal displacement of the bit without rotation allows mounting of sensors for real-time analysis of the drilled medium. The combination of sampling probing and sensing allows the USDC to be used as a lab-on-a-drill system.

\subsection{USDC with integrated sensor suite}

Since the USDC bit does not turn and its vibration amplitude is relatively small one can easily mount sensors on the bit and conduct real-time tests during drilling or coring. Two types of sensors were successfully demonstrated to date: thermocouple and fiberoptic. A thermocouple was used to measure the rate and maximum rise of temperature and these values were found to correlate to the hardness of the rock being drilled. Even though these thermal variables are dependent on the heat conductivity and capacity of the drilled object, one can assume with a reasonable accuracy that most rocks have thermal properties within a comparatively narrow range. Compiling 
temperature rise rate and maxima as a function of time for variety of drilled materials has demonstrated the feasibility of using a thermocouple-on-the-bit as a means of assessing the drilled medium hardness (see Figure 2).

Other tests using an optical fiber that was approximately $160 \mu \mathrm{m}$ in diameter was imbedded into 10 -mm diameter coring bit with a 1-mm wall thickness. Reflection data in the wavelength range of 400-1200 nm were recorded. The current study is focusing on the use of fiberoptics with UV light in the range of 200-nm wavelength and at this range it is easier to determine if biological markers are present in the drilled medium.

\begin{tabular}{|l|l|}
\hline $\begin{array}{c}\text { Soil conditions and/or } \\
\text { Rock Type }\end{array}$ & \multicolumn{1}{|c|}{$\begin{array}{c}\text { Young's } \\
\text { Modulus }\end{array}$} \\
\hline Loose sand & $10-25 \mathrm{MPa}$ \\
\hline Medium dense sand & $20-60 \mathrm{MPa}$ \\
\hline Dense sand & $50-100 \mathrm{MPa}$ \\
\hline Sedimentary sandstone & $10-60 \mathrm{GPa}$ \\
\hline Igneous Basalt & $60-80 \mathrm{GPa}$ \\
\hline Sedimentary limestone & $60-80 \mathrm{GPa}$ \\
\hline Igneous Anorthosite & $83 \mathrm{GPa}$ \\
\hline
\end{tabular}

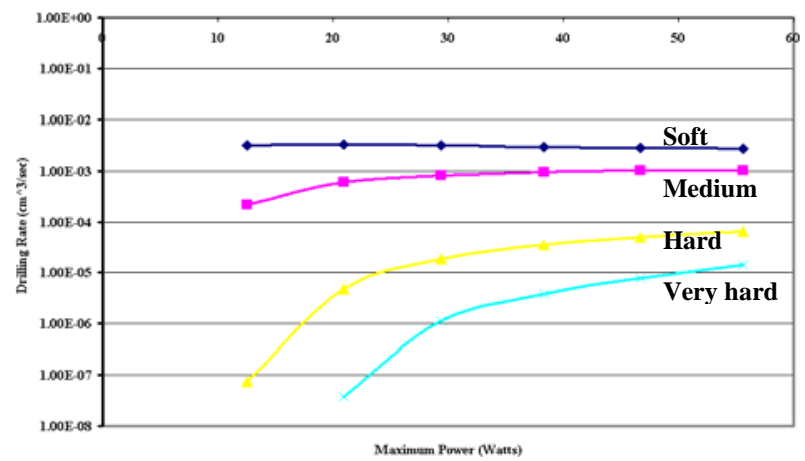

TABLE 1: Typical values of the elastic modulus for soils and rocks

FIGURE 1: Analytical drilling rates in rocks with various hardness levels using $10 \mathrm{~W}$ average power. The rocks are classified by their compression strength, where: Soft: $0-50$; Medium: $50-$ 100; Hard: $100-200$; and Very hard: >200 (MPa).
FIGURE 2: The measured temperature maxima and initial temperature rate as a function of hardness (inverse drilling rate) for variety of media.

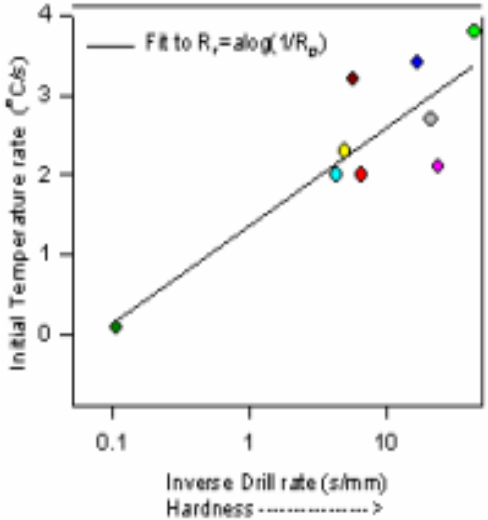

- Packed Sand

- BesaltSceria

- Bridk

- Biehops Tut

- Limestone

- Vesicular Basat

- Volcanic Lkthic Conglomerate

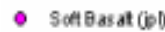

- Olwhe Bast

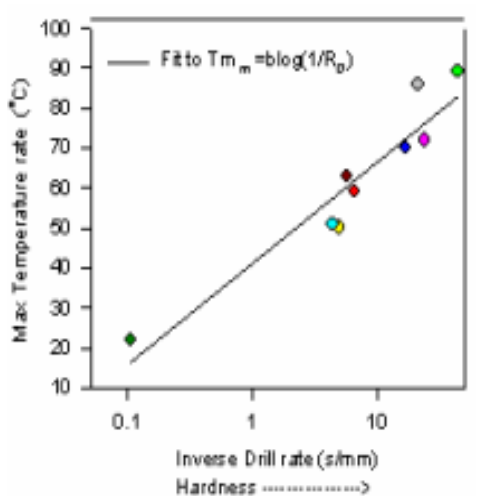

\section{ULTRASONIC/SONIC GOPHER}

The U/S Gopher is a large scale version of the USDC where a coring bit is used having a diameter that is great 
than the actuator. The formed core is removed from the borehole and the gopher is reeled back into the borehole to create a core from the new depth (see schematic view in Figure 3). The developed gopher is being designed to supercede any prior drilling techniques in reaching as deep as 20-meters at Lake Vida, Antarctica, reaching the brine section that is below the 16-m depth ice. Early versions of the U/S gopher indicated that coring through $20^{\circ} \mathrm{C}$ ice may prove a bigger challenge than coring through rock. In our recent study we addressed such issues as chip handling, core breaking and ice melting during drilling. The later is the result of the concern of getting the bit jammed due to refreezing. Test of the USDC operation in low temperature as a function of time is shown graphically in Figure 4.

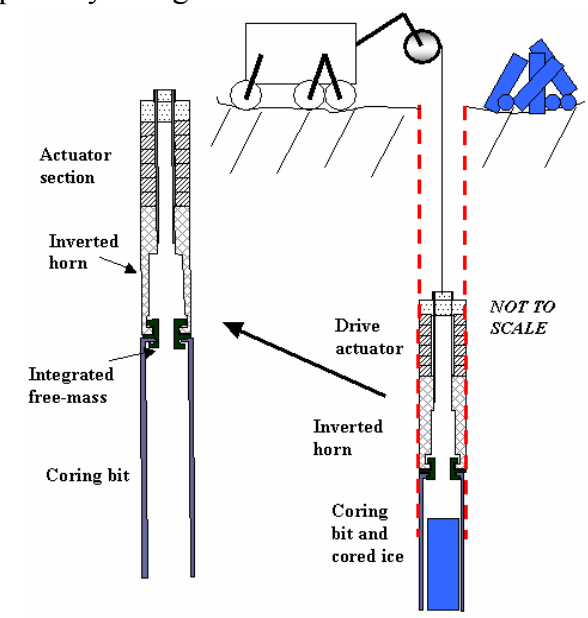

FIGURE 3: Schematic view of the ultrasonicgopher operating inside the borehole.

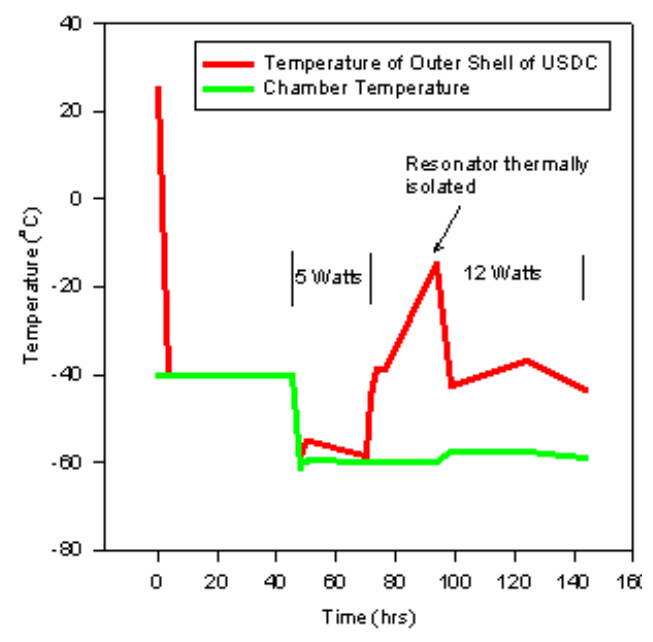

FIGURE 4: Tests of the USDC operation at low temperatures (conducted at the JPL's Extraterrestrial Materials Simulation Laboratory)

\section{PACKED SOIL PENETRATOR}

Recent, a challenged was posed to penetrate packed soil to penetrate about 1 meter using $1 / 8$ " - 3/16" diameter probes via low axial load. The use of push rod requires a force of several hundreds pounds when using and easily results in buckling of the probes. A novel Ultrasonic/Sonic Impacting Penetrator (USIP) was developed and demonstrated to greatly reduce the required push force. This device uses an ultrasonic transducer that energizes a free-mass to impact a rod probe, which consists of a loading-shaft and a bit. The impact of the free-mass through a shoulder on the rod probe creates an impulse wave. This wave propagates to the tip of the probe and significantly enhances the rate of penetration as well as reduces the required push force compared to an unaided rod. In demonstration of the USIP capability it was shown that the required push force to penetrate highly packed soil down to about 1-meter was reduced from $200 \mathrm{lb}$ to $7 \mathrm{lb}$. A graphic view of the developed unit is shown in Figure 5 .

To optimize the components of the USIP, we used a numerical analysis program that we recently developed. This effort involved both modal and impact analysis, where the modal analysis was used to determine the dimensions of the horn and backing for a given resonance frequency in the required range. We also used the results of this analysis to adjust the dimension of the ultrasonic horn so that the neutral plane matches the mounting plane to avoid effects of the transducer vibration on the actuator support structure. The impact analysis, which determines the interaction between the free-mass and the ultrasonic horn, was used to derive an optimal weight of the free-mass.

\section{U/S ANCHOR}

Increasingly, NASA missions are involved with in-situ exploration tasks where anchoring is becoming a critical aspect of the mission. The use of an anchor that can be easily mounted and extracted while requiring very low axial force is critical for missions to planets with a very low gravity. Such a capability to anchor via low axial load using low mass device and relatively low power is needed to support such NASA platforms as legged and wheeled rovers as well as inflatable structures and landers that would operate in low g environments and explore possible water rich environments including canyon walls. Also, it can used to support astronauts who may need to climb steep mountains as part of future exploration by humans in rugged extraterrestrial terrain. 
A U/S Anchor was designed and fabricated using a modification of the USDC. The device takes advantage of the USDC ability to operate under low axial force, low average power, via lightweight mechanism. Using the drilling capability the USDC, the device was turned into an anchoring mechanism by drilling in an angle with the normal to the surface. Each leg of the climbing robot can be instrumented with such an anchor. Operating the hammering action of the USDC in reverse allows its extraction from the medium onto which it was anchored and avoids possible jamming.

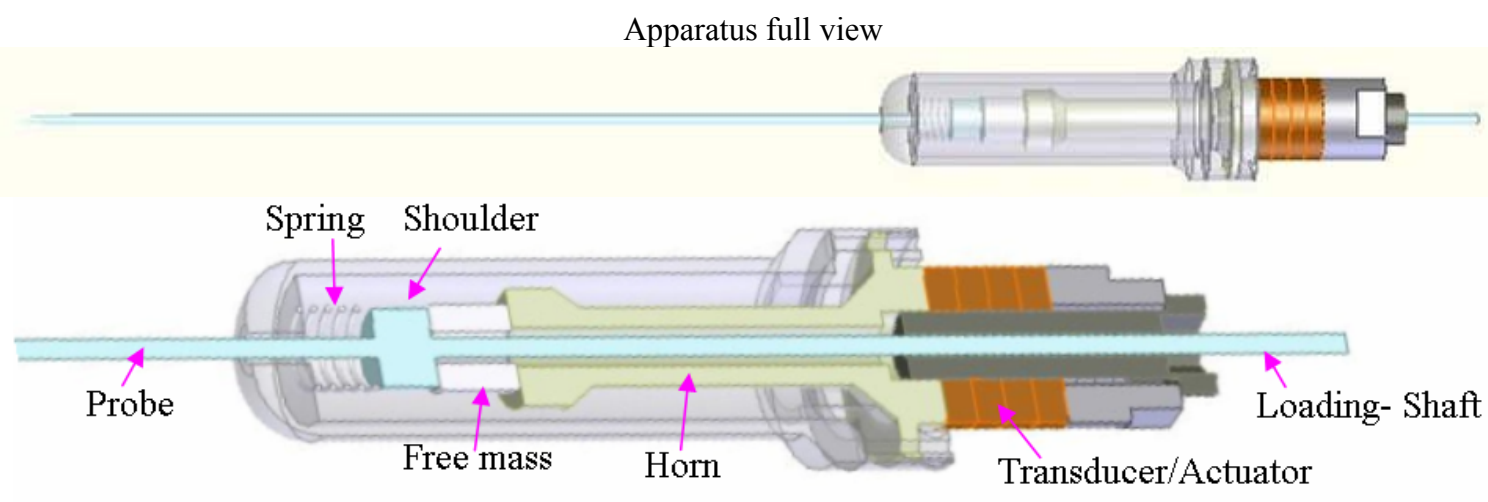

FIGURE 5: A schematic view of the disclosed USIP.

\section{USDC WITH VARIOUS NOVEL HORNS}

The horn is an important part of the USDC actuator amplifying the produced vibration amplitude. Generally, the horn is made as a step down diameter from the one that the piezoelectric stack has and thus gaining from the relative surface areas reduction.

\subsection{Dog-bone shape horn}

Recently, a dog-bone design was introduced and demonstrated improvement of the drilling performance as well as a benefit for mounting bits as well as operating with dual free-mass to allow reverse operation too. For this purpose, various horn designs were examined analytically and compared to conventional and solid shape horns and analytically it was shown that the dog-bone design has a superior performance. To demonstrate the capability of the dog bone horn a finite element modeling was used to determine the control parameters and showed the excitation of enhanced tip displacement and velocity. A view of the U/S gopher using the dog bone horn is shown in Figure 6 (bottom of the item on the right). The other components of the new Gopher are also shown including the bit for drilling ice at $-20^{\circ} \mathrm{C}$ (shown on the left) and the actuator (top of the item on the right) coated by Teflon sleeve. A view of the dual free-mass design is shown graphically in Figure 7.

FIGURE 6: A view of the new USG with a dog bone horn.

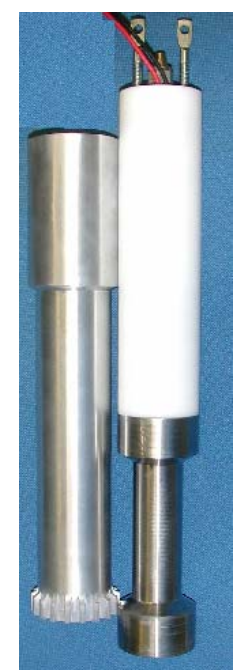




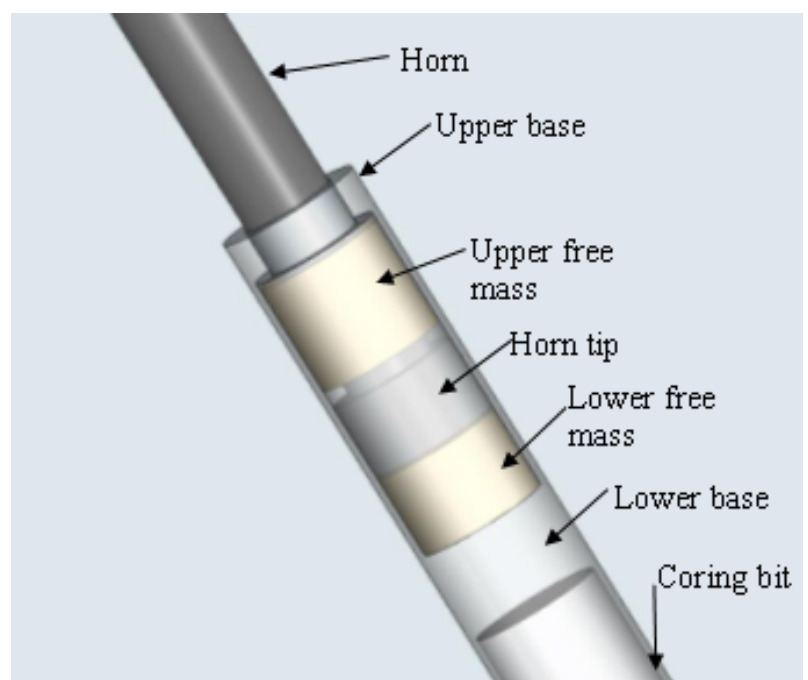

FIGURE 7: A view of a dog bone horn with upper and lower free mass for forward and reverse hammering.

\subsection{Folded horn}

The length of the horn in the USDC actuator can be a concern when there are space concerns and reduction of its size is can be critical to the integration of the unit as a sampler in future missions. A novel horn was developed with hollow configuration that amplifies vibrations of high power actuation mechanisms as shown in Figure 8 . The folded horn can be configured in axis-symmetric and planar shapes to provide manufacturing options. In addition the use of reflectors at the folds allows for control of the phase of the reflected strain wave and for the introduction of constructive bending vibrations that enhance the amplification of the actuation.

FIGURE 8: A schematic view of the cross section of a folded horn

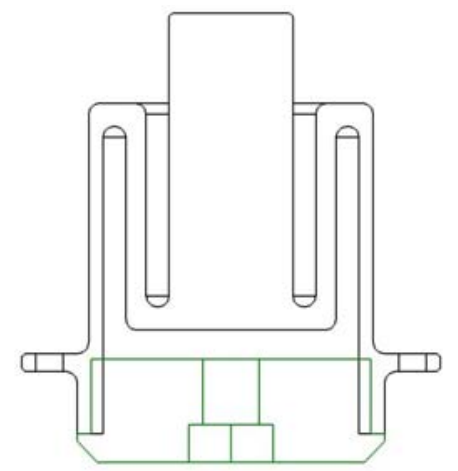

\section{USDC WITH NOVEL BITS}

\subsection{Ultrasonic/Sonic Rock Abrasion Tool (URAT)}

Abrasion of the surface of a rock using low axial force and limited average power is considered a critical technology for future NASA planetary exploration missions. Conventional rotating mechanisms require high axial loads and they are involved with contamination sources such as lubricants and ground filings from their motor gearbox. The use of the USDC offers significant advantages in requiring low axial force, low average power, low number of components, and the capability to produce a mechanism of removal of powered cuttings from the borehole. In its original form, the USDC was designed to drill or core and it is not was not designed to remove layers of weathered material from rocks. To address the need for an abrasion tool that allows for the removal of weathered layers from rock surfaces the Ultrasonic Rock Abrasion Tool (URAT) was developed. An abrasion bit was designed similar to the hammering surface of a meat tenderize meat and has been dubbed the "tenderizer." Teeth were machined on the bottom of the disk that is part of the abrasion bit. These teeth amplify the drilling pressure and enhance the action of the URAT. A schematic cross-section drawing is shown in Figure 9 illustrating the components and the compact structure of the URAT. A view of abraded basalt is shown in Figure 10. The abrasion tool consists of a 1.6 in. $(40-\mathrm{mm})$ diameter disk that is attached to a shank that fits around the horn. The 
free-mass is placed inside the shank between the horn and the bottom of hole along the inner part of the bit. On the bottom of the disk teeth were machined in the form of pins that sticks out of the disk.

(URAT)

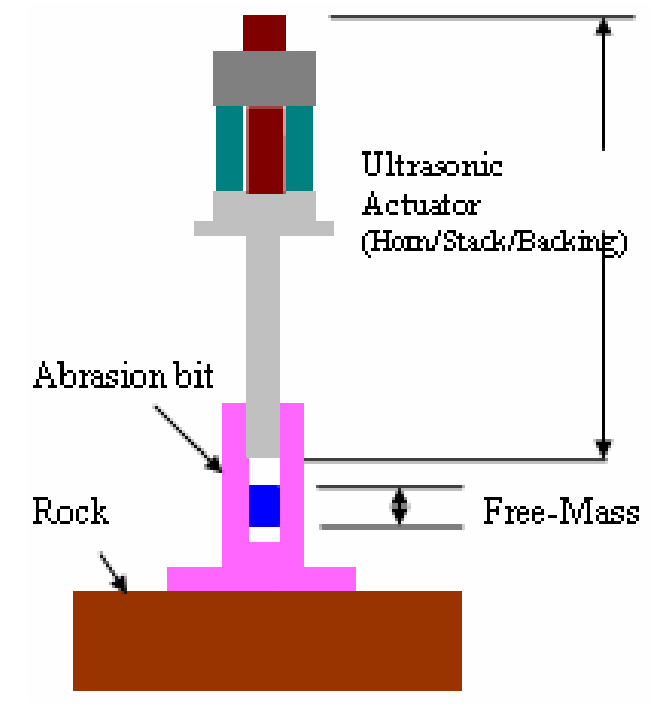

FIGURE 10: Photographic view of a basalt that was abraded by the URAT. The footprint of the abrasion bit can be seen on the surface.

\subsection{Interchangeable bit}

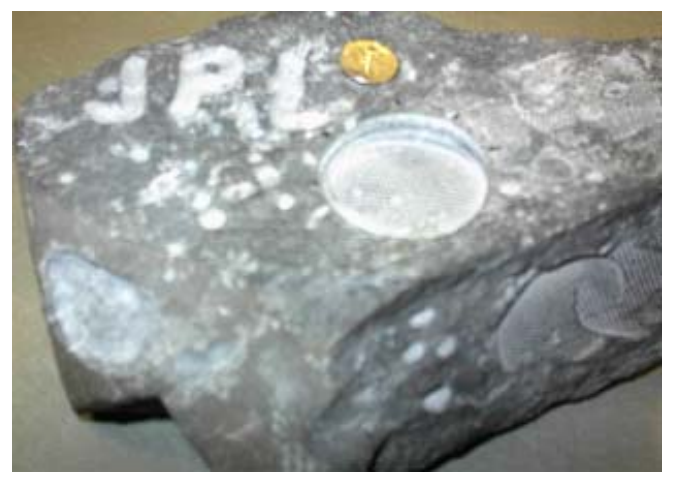

One of the most promising benefits of the USDC is the simple interface it provides to the bit, and the simplicity of the shape of the bit itself. The use of multiple bits is essential as it is simply not practical to accomplish the multitude of tasks envisioned for future missions with a single tool. These tasks can, however, be accomplished by a single actuator that utilizes multiple bits for drilling, coring, surface preparation, and sampling. The USDC makes multiple bits and the exchange of those bits a very practical idea. Many of the bits are as simple as a tube of a single material. The bit does not require sharpening, and, if a bit where to get damaged for whatever reason, it could simply be replaced.

\subsection{All in one bit}

The use of multiple bits requires a manipulation system that allows exchanging bits as needed. If a manipulation system is not available it is highly desirable accomplish as many functions as possible using a single bit. For this purpose an all-in-one bit was developed and demonstrated. The bit is shown in Figure 11 and it consists of a tube with a wedge at the top of the inner surface of the bit, a set of springs near the tip and a push rod that is inserted thru a center hole in the bit. Once a core is produced at a length of the bit, the wedge introduces transverse forces at the top of the core to cause maximum stress near the root and shear fracture. The side springs hold the produced core from removable from the borehole and the core is extracted when needed by using the push rod from the top of the bit.

FIGURE 11: A schematic view of the core extraction bit.

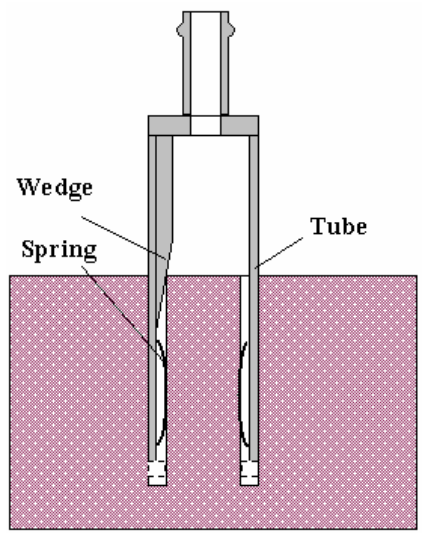




\section{SUMMARY}

The ultrasonic/sonic driller/corer (USDC) is investigated for various potential planetary applications. To allow effective design and construction of the various modifications of the USDC an analytical model was developed to predict its behavior towards the goal of optimizing its performance. Physical models were developed for each section of the device and their interactions. The developed models were integrated to allow investigation of the various interactions of the USDC and effective designs to support various applications. Various designed were developed and demonstrated including the Lab-on-a-Drill, Ultrasonic Gopher, soil penetrator and many others and various configurations of the horn and the bit were used to provide multifuncitonality. The Lab-on-a-Drill is intended to take advantage of the probing capabilities of the USDC, the capability to sample cores and powdered cuttings as well as the fact that sensors can be easily mounted on the bit and allow real time data acquisition while drilling. The URAT was demonstrated to remove rock layers from as hard as Basalt. The Ultrasonic Gopher operates in a cyclic mode of coring, uploading, core caching and downloading. This device is being investigated for drilling up to 20-meters in ice in Antarctica. The potential of the USDC technology is continuing to be investigated with goal of using its various configurations in future missions.

\section{ACKNOWLEDGEMENT}

Research reported in this manuscript was conducted at the Jet Propulsion Laboratory (JPL), California Institute of Technology, under a contract with the National Aeronautics and Space Administration (NASA).

\section{REFERENCES}

Bao X., Y. Bar-Cohen, Z. Chang, B. P. Dolgin, S. Sherrit, D. S. Pal, S. Du, and T. Peterson, IEEE Transaction on Ultrasonics, Ferroelectrics and Frequency Control (UFFC), Vol. 50, No. 9, (Sept. 2003), pp. 1147-1160.

Bao X., Y. Bar-Cohen, Z. Chang, S. Sherrit and R. Stark, "Ultrasonic/Sonic Impacting Penetrator (USIP),” NTR Docket No. 41666 (December 22, 2004).

Bar-Cohen Y., and S. Sherrit, "Self-Mountable and Extractable Ultrasonic/Sonic Anchor (U/S-Anchor)," NTR Docket No. 40827 (December 9, 2003c).

Bar-Cohen Y., and S. Sherrit, "Thermocouple-on-the-bit a real time sensor of the hardness of drilled objects," NTR Docket No. 40132 (February 1, 2003a)

Bar-Cohen Y., J. Randolph, C. Ritz, G. Cook and X. Bao, and S. Sherrit, "Sample Preparation, Acquisition, Handling and Delivery (SPAHD) System using the Ultrasonic/Sonic Driller/Corer (USDC) with Interchangeable Bits," NTR Docket No. 30640 (May 1, 2002).

Bar-Cohen Y., S. Sherrit and J. L. Herz "Ultrasonic/Sonic Jackhammer (USJ)," NTR Docket No. 40771 (Oct. 31 , 2003b).

Bar-Cohen Y., S. Sherrit, B. Dolgin, S. Askin, T. M. Peterson, W. Bell, J. Kroh, D. Pal, R. Krahe, and S. Du, "Ultrasonic/Sonic Mechanism of Deep Drilling (USMOD)," NTR, Docket No. 30291, (July 17, 2001)

Bar-Cohen Y., S. Sherrit, B. Dolgin, T. Peterson, D. Pal and J. Kroh, "Ultrasonic/Sonic Driller/Corer (USDC) With Integrated Sensors," NASA New Technology Report (NTR), Docket No. 20856 (August 30, 1999)

Dolgin B., S. Sherrit, Y. Bar-Cohen, R. Rainen, S. Askins and D. Sigel, D. Bickler, J. Carson, S. Dawson, X. Bao, and Z. Chang, and T. Peterson, "Ultrasonic Rock Abrasion Tool (URAT)," NTR Docket No. 30403 (Oct. 12, 2001b).

Dolgin B., S. Sherrit, Y. Bar-Cohen, S. Askins, D. Sigel, X. Bao, and Z. Chang, "Ultrasonic/ Sonic Vibrating/Rotating Tool” NTR Docket No. 30370 (Sept. 5, 2001a)

Sherrit S., S. A. Askins, M. Gradziel, B. P. Dolgin, Y. Bar-Cohen, X. Bao, and Z. Cheng, "Novel Ultrasonic Horns for power ultrasonics, "NTR Docket No. 30489 (Dec. 6, 2001).

Sherrit S., Y. Bar-Cohen, B. Dolgin, X. Bao, and Z. Chang, "Ultrasonic Crusher for Crushing, Milling, and Powdering," NTR Docket No. 30682 (June 21, 2002).

Sherrit S., Y. Bar-Cohen, X. Bao, Z. Chang, D. Blake and C. Bryson, "Ultrasonic/Sonic Rock Powdering Sampler and Delivery Tool," NTR Docket No. 40564 (August 13, 2003) 